\title{
Blow-Up of Solutions for Wave Equation Involving the Fractional Laplacian with Nonlinear Source
}

\author{
Y. Bidi, ${ }^{1}$ A. Beniani, ${ }^{2}$ M. Y. Alnegga $\left(D,{ }^{3}\right.$ and A. Moumen ${ }^{4}{ }^{4}$ \\ ${ }^{1}$ University of Djillali Liabes - B. P. 89, Sidi Bel Abbes 22000, Algeria \\ ${ }^{2}$ Department of Mathematics, University of Ain Temouchent, \\ Laboratory of Analysis and Control of Partial Differential Equations, Ain Temouchent 46000, Algeria \\ ${ }^{3}$ Department of Mathematics, College of Sciences and Arts, Qassim University, Ar-Rass, Saudi Arabia \\ ${ }^{4}$ Department of Mathematics, Faculty of Science, University of Hail, Hail 55425, Saudi Arabia
}

Correspondence should be addressed to M. Y. Alnegga; mnkaaa@qu.edu.sa

Received 22 August 2021; Revised 13 September 2021; Accepted 17 September 2021; Published 30 September 2021

Academic Editor: Muhammad Irfan

Copyright (c) $2021 \mathrm{Y}$. Bidi et al. This is an open access article distributed under the Creative Commons Attribution License, which permits unrestricted use, distribution, and reproduction in any medium, provided the original work is properly cited.

In this paper, we study the blow-up of solutions for wave equation involving the fractional Laplacian with nonlinear source.

\section{Introduction and Brief History of Fractional Integrodifferentiation}

Let $\Omega \subset \mathbb{R}^{n}, n \geq 1$ be an open domain with Lipschitz boundary $\partial \Omega$. In this article, we consider the hyperbolic initial-boundary value problem involving the fractional Laplacian; for $w=w(x, t)$, we consider the wave equation with power nonlinearity:

$$
\begin{cases}\partial_{t}^{2} w+(-\Delta)^{r} w+(-\Delta)^{r} \partial_{t} w=w|w|^{p-2}, & x \in \Omega, t>0, \\ w=0, & x \in \partial \Omega, t>0, \\ w(x, 0)=w_{0}(x), \partial_{t} w(x, 0)=w_{1}(x), & x \in \Omega,\end{cases}
$$

where $(-\Delta)^{r}$ is the fractional Laplacian such that $r \in(0,1)$. The exponent $p$ satisfies

$$
2<p \leq \frac{2 n}{n-2 r}=2_{r}^{*}, \quad n>2 r .
$$

The fractional integrodifferentiation operation is a generalization of the differentiation operations. The idea of fractional differentiation as a generalization of the concept of the derivative to the noninteger value of $a$ arose almost simultaneously with the very concept of differentiation. The first mention of this idea occurs in the correspondence of
G. W. Leibniz and Marquis de l'Hospital in 1695 (see [1]). The idea of fractional integrodifferentiation was further developed in the works of L. Euler, who in 1738 noticed that an expression can be given meaning even for noninteger values (see [2]). An explicit calculation formula was given in the treatise by S. Lacroix in 1820 (see [3]). Also in 1812, P.S. Laplace put forward the idea of the possibility of differentiating noninteger order for some functions. The first definition of the derivative of noninteger order was given by J. Fourier in 1822. In its modern form, fractional integrodifferentiation was formed in the works of N.H. Abel and J. Liouville. In 1823, in connection with the problem of tautochrone-a curve, when sliding along which, under the influence of gravitational forces, a body reaches its lowest point in the same time, regardless of its initial position. The idea of considering fractional differentiation as an operation inverse to fractional integration was first proposed by Holmgren in 1865 (see [4-6]). A year later, Grunwald, who was not familiar with Holmgren's work, came to the same idea of Letnikov in 1868 (see [7-13]).

In [14], an efficient novel technique, namely, the q-homotopy analysis transform method (q-HATM), is applied to find the solution for the time-fractional Kaup-Kupershmidt (KK) equation and the study of fractional Emden-Fowler (FEF) equations by utilizing a new 
adequate procedure; specifically, the q-homotopy analysis transform method (q-HATM) is considered in [15].

Fractional wave systems with continuous nonlinearities are possessed by a large number of researchers. In [16], the authors considered initial-boundary value problem of degenerate Kirchhoff-type

$$
\partial_{t}^{2} w+[w]_{r}^{2(\theta-1)}(-\Delta)^{r} w=|w|^{p-1} w \text {, in } \Omega \times \mathbb{R}_{+},
$$

where $\Omega \subset \mathbb{R}^{n}, n \geq 1$ is a bounded domain with Lipschitz boundary, $\theta \in\left[1,2^{*}\right]$, and $[w]_{r}$ is the Gagliardo seminorm of $w$ defined by

$$
[w]_{r}=\left(\int_{\Omega} \int_{\Omega} \frac{|w(x)-w(z)|^{2}}{|x-z|^{n+2 r}} \mathrm{~d} x \mathrm{~d} z\right)^{1 / 2} .
$$

The authors obtained, under appropriate conditions, the global existence in time and finite blow-up of solutions for (3) owing to the Galerkin method combined with the potential wells. They also showed the global existence of solutions under critical initial conditions. In [17], the authors studied the following damped degenerate Kirchhoff equation:

$$
\begin{gathered}
\partial_{t}^{2} w+[w]_{r}^{2(\theta-1)}(-\Delta)^{r} w+\left|\partial_{t} w\right|^{\alpha-1} \partial_{t} w \\
+w=|w|^{p-2} w, \text { in } \Omega \times \mathbb{R}_{+},
\end{gathered}
$$

where $2<\alpha<2 \theta<p<2^{*}<r$. The global existence, behavior of solutions, and blow-up in time for (4) are obtained, under appropriate assumptions. In [18], the IBVP of Kirchhoff wave equation is considered. Under some sufficient conditions, the blow-up in finite time is shown by using a modified concavity method; for more details, see [19-27].

$$
\partial_{t}^{2} w+[w]_{r}^{2(\theta-1)}(-\Delta)^{r} w=|w|^{p-2} w \text {, in } \Omega \times \mathbb{R}_{+} .
$$

We highlight here the novelty of the problem:

(1) It is interesting to note that simultaneously with the theoretical developments of classical nonlinear wave operation, practical applications of fractional integrodifferentiation operation can also be found

(2) It is shown that when the nonlinear source dominates the fractional Laplacian in (2), this ensures the global nonexistence in time (blow-up) of solutions

(3) Our results extend many recent results in the literature

\section{Auxiliary Results and Function Spaces}

The fractional Laplacian $(-\Delta)^{r} w$ of the function $w$ is given by

$$
(-\Delta)^{r} w(x)=C \int_{\mathbb{R}^{n}} \frac{w(x)-w(z)}{|x-z|^{n+2 r}} d z, \quad \forall x \in \mathbb{R}^{n},
$$

where

$$
C^{-1}=\int_{\mathbb{R}^{n}} \frac{1-\cos \left(\zeta_{1}\right)}{|\zeta|^{n+2 r}} \mathrm{~d} \zeta .
$$

We define the fractional-order Sobolev space by

$$
W^{r, 2}(\Omega)=\left\{v \in L^{2}(\Omega): \int_{\Omega} \int_{\Omega} \frac{|v(x)-v(z)|^{2}}{|x-z|^{n+2 r}} \mathrm{~d} x \mathrm{~d} z<\infty\right\},
$$

equipped with the norm

$$
\|w\|_{W^{r, 2}(\Omega)}=\left(\int_{\Omega}|w|^{2} \mathrm{~d} x+\int_{\Omega} \int_{\Omega} \frac{|v(x)-v(z)|^{2}}{|x-z|^{n+2 r}} \mathrm{~d} x \mathrm{~d} z\right)^{1 / 2} .
$$

Let

$$
W_{0}^{r, 2}(\Omega)=\left\{w \in W^{r, 2}(\Omega): w=0, x \in \partial \Omega\right\},
$$

be a closed linear subspace of $W^{r, 2}(\Omega)$, and its norm is given by

$$
\|w\|_{W_{0}^{r, 2}(\Omega)}=\left(\int_{\Omega} \int_{\Omega} \frac{|v(x)-v(z)|^{2}}{|x-z|^{n+2 r}} \mathrm{~d} x \mathrm{~d} z\right)^{1 / 2} .
$$

The space $W_{0}^{r, 2}(\Omega)$ is a Hilbert space with inner product

$$
\langle w, u\rangle_{W_{0}^{r, 2}(\Omega)}=\int_{\Omega} \int_{\Omega} \frac{(w(x)-w(z))(u(x)-u(z))}{|x-z|^{n+2 r}} \mathrm{~d} x \mathrm{~d} z .
$$

\section{The Potential Wells}

For simplicity, in this section, we consider problem (1) in stationary case. In fact, if we replace $w$ in this section by $w(t)$ for any $t \in[0, T)$, all the facts are still valid. We define

$$
\mathscr{J}(w)=\frac{1}{2}\|w\|_{W_{0}^{r, 2}(\Omega)}^{2}-\frac{1}{p}\|w\|_{p}^{p} .
$$

We denote

$$
\mathscr{I}(w)=\|w\|_{W_{0}^{r, 2}(\Omega)}^{2}-\|w\|_{p}^{p} .
$$

We introduce now the stable set as follows:

$$
\mathscr{W}=\left\{w \in W_{0}^{r, 2}(\Omega): \mathscr{I}(w)>0, \mathscr{J}(w)<d\right\} \cup\{0\},
$$

where the mountain pass level $d$ is defined as

$$
d=\inf _{w \in W_{0}^{r, 2}(\Omega) \backslash\{0\}}\left\{\sup _{\mu \geq 0} \mathscr{J}(\mu w)\right\} .
$$

We introduce the so-called Nehari manifold:

$$
\mathscr{N}=\left\{w \in W_{0}^{r, 2}(\Omega) \backslash\{0\}: \mathscr{I}(w)=0\right\} .
$$

Then potential depth $d$ is characterized by

$$
d=\inf _{w \in \mathcal{N}} \mathscr{F}(w)
$$

which implies that

$$
\operatorname{dist}(0, \mathcal{N})=\min _{w \in \mathcal{N}}\|w\|_{W_{0}^{r, 2}(\Omega)} .
$$

We will prove the invariance of the set $\mathscr{W}$. 
For the reader's convenience, we recall the main embedding results for the fractional Sobolev spaces; see [28] for details.

Lemma 1. Let $\Omega$ be bounded domain. Then

(1) The embedding $W_{0}^{r, 2}(\Omega) \hookrightarrow L^{p}(\Omega)$ is compact for any $p \in\left[1,2_{r}^{*}\right)$

(2) The embedding $W_{0}^{r, 2}(\Omega) \hookrightarrow L^{2_{r}^{*}}(\Omega)$ is continuous

\section{Lemma 2}

(1) For any $s \in\left[1,2_{r}^{*}\right]$, there exists a positive constant $C_{0}=C_{0}(n, s, r)$ such that for any $u \in W_{0}^{r, 2}(\Omega)$

$$
\|w\|_{L^{s}(\Omega)} \leq C_{0} \int_{\Omega} \int_{\Omega} \frac{|u(x)-u(z)|^{2}}{|x-y|^{n+2 r}} \mathrm{~d} x \mathrm{~d} y .
$$

(2) For any $s \in\left[1,2_{r}^{*}\right]$ and any bounded sequence $\left(u_{j}\right)_{j}$ in $W_{0}^{r, 2}(\Omega)$, there exists $u$ in $L^{s}\left(\mathbb{R}^{n}\right)$, with $u=0$ a.e. in $\mathbb{R}^{n}-\Omega$, such that up to a subsequence, still denoted by $\left(u_{j}\right)_{j}$

$$
u_{j} \longrightarrow u \text { strongly in } L^{s}(\Omega) a s j \longrightarrow \infty .
$$

Definition 1. A function $w=w(x, t)$ is said to be a global (weak) solution of problem (1), if

$$
\begin{array}{r}
w \in L^{\infty}\left(0, \infty, W_{0}^{r, 2}(\Omega)\right), w_{t} \in L^{\infty}\left(0, \infty, L^{2}(\Omega)\right), \\
w_{o} \in L^{\infty}\left(0, \infty, W_{0}^{r, 2}(\Omega)\right), w_{t} \in L^{\infty}\left(0, \infty, L^{2}(\Omega)\right) \\
w_{1} \in L^{\infty}\left(0, \infty, L^{2}(\Omega)\right),
\end{array}
$$

and for any $\phi \in L^{\infty}\left(0, \infty, W_{0}^{r, 2}(\Omega)\right), t \in \mathbb{R}_{+}^{*}$,

$$
\begin{aligned}
& \left(w_{t}(., t), \phi(., t)\right)+\frac{1}{2} \int_{0}^{t}\left(w_{t}(., t), \phi(., t)_{W_{0}^{r, 2}(\Omega)}\right) \mathrm{d} \tau+\int_{0}^{t}\left(w_{t}(., t), \phi(., t)_{W_{0}^{r, 2}(\Omega)}\right) \mathrm{d} \tau \\
= & \left(w_{1}, \phi(., 0)\right)+\int_{0}^{t}\left(w(., \tau)|w(., \tau)|^{p-2}, \phi(., \tau)\right) \mathrm{d} \tau .
\end{aligned}
$$

If a (weak) global solution $w$ belongs to $C(0, \infty$; $W_{0}^{r, 2}(\Omega)$ ), we say that $u$ is a strong global solution of problem (1).

The energy $\mathscr{E}$ of solution at time $t$ to (1) is given by

$$
\mathscr{E}(t)=\frac{1}{2}\left\|\partial_{t} w(t)\right\|_{2}^{2}+\mathscr{J}(w) .
$$

Lemma 3. Let $w(x, t)$ be a weak solution of problem (1). If $w_{0} \in \mathscr{W}, w_{1} \in L^{2}(\Omega)$, then $\mathscr{E}(t) \leq \mathscr{E}(0)$.

\section{Blow-Up Result}

In this section, we prove the blow-up result to problem (1)

Lemma 4. Let $w(x, t)$ is the weak solution of problem (1). If $w_{0} \in \mathscr{W}$ and $w_{1} \in L^{2}(\Omega)$ satisfying that

$$
\begin{aligned}
\|w\|^{2} & \geq \frac{2 p}{p-2} K_{\mathscr{C}}(0), \\
\left(\partial_{t} w, w\right)_{W_{0}^{r, 2}(\Omega)} & <0, \\
w_{0} & \in \mathscr{M}, \\
\int_{\Omega} w_{0} w_{1} \mathrm{~d} x & >0,
\end{aligned}
$$

then any solution of (1) belongs to $\mathscr{M}$.
Proof. We claim that $w \in \mathscr{M}$ for $t \in[0, T)$; by contradiction, we suppose that $t_{0} \in(0, T)$ is the first time such that

$$
\begin{gathered}
I\left(w\left(t_{0}\right)\right)=0, \\
\mathscr{I}(w(t))<0 \text { for } t \in\left[0, t_{0}\right) .
\end{gathered}
$$

We first introduce an auxiliary function,

$$
M(t)=\|w\|^{2},
$$

and directly

$$
\begin{aligned}
M^{\prime}(t) & =\left(\partial_{t} w, w\right)+\left(w, \partial_{t} w\right)=2\left(\partial_{t} w, w\right), \\
M^{\prime \prime}(t) & =2\left(\partial_{t}^{2} w, w\right)+2\left\|\partial_{t} w\right\|^{2} .
\end{aligned}
$$

Multiplying (1) 1 by $w$ and then by integration over $\mathbb{R}^{n}$, we have

$$
\begin{aligned}
& (\partial) t^{2} w, w+(w, w) W^{r, 2} 0(\Omega)+(\partial t) w, w W^{r, 2} 0(\Omega) \\
= & \int \Omega w^{p-2} w \mathrm{~d} x,
\end{aligned}
$$

so that

$$
\left(\partial t^{2} w, w\right)=-\|w\|_{W_{0}^{r, 2}(\Omega)}-\left(\partial_{t} w, w\right)_{W_{0}^{r, 2}(\Omega)}+\int_{\Omega} w|w|^{p-2} w \mathrm{~d} x .
$$

Substituting (27) into (36), we obtain 


$$
\begin{aligned}
M^{\prime \prime}(t)= & \left.2\left\|\partial_{t} w\right\|_{W}^{r, 2} 0(\Omega)-2 w_{W^{r, 2}} 0(\Omega)-2 \partial_{t} w, w\right)_{W^{r, 2}} 0(\Omega) \\
& +2 \int_{\Omega} w \mid w^{p-2} w \mathrm{~d} x .
\end{aligned}
$$

By (27), we have

$$
M^{\prime \prime}(t) \geq 2\left\|\partial_{t} w\right\|^{2}-2 \mathscr{I}(w) .
$$

By (31), we have $M^{\prime \prime}(t)>0$ for any $t \in\left[0, t_{0}\right)$; then, $M^{\prime}(t)$ is strictly increasing on $\left[0, t_{0}\right)$. Thus

$$
M^{\prime}(t)>M^{\prime}(0)>0 \text { for } t \in\left[0, t_{0}\right) .
$$

We have $M(t)$ is also strictly increasing on $\left[0, t_{0}\right)$. We have

$$
M(t)>M(0) \geq \frac{2 p}{p-2} K \mathscr{E}(0) \text { for all } t \in\left[0, t_{0}\right) .
$$

From the continuity of $w$ at $t=t_{0}$, it follows that

$$
M\left(t_{0}\right)=\left\|w\left(t_{0}\right)\right\|^{2}>2 \frac{p}{p-2} K \mathscr{E}(0) .
$$

On the other hand,

$$
\begin{aligned}
\mathscr{E}(0) & \geq \mathscr{E}(t)=\frac{1}{2}\left\|\partial_{t} w\right\|_{2}^{2}+\frac{1}{2}\|w\|_{W_{0}^{r, 2}(\Omega)}^{2}-\frac{1}{p}\|w\|_{p}^{p} \\
& =\frac{1}{2}\left\|\partial_{t} w\right\|_{2}^{2}+\left(\frac{1}{2}-\frac{1}{p}\right)\|w\|_{W_{0}^{r, 2}(\Omega)}^{2}+\frac{1}{p} \mathscr{I}(w) .
\end{aligned}
$$

Together with (30) and Lemma 2, we get

$$
\begin{aligned}
\mathrm{E}(0) & \geq \frac{1}{2}\left\|\partial_{t} w\left(., t_{0}\right)\right\|_{2}^{2}+\left(\frac{1}{2}-\frac{1}{p}\right)\left\|w\left(., t_{0}\right)\right\|_{W_{0}^{r, 2}(\Omega)}^{2} \\
& \geq \frac{1}{2}\left\|\partial_{t} w\left(., t_{0}\right)\right\|_{2}^{2}+\frac{p-2}{2 p} K^{-1}\left\|w\left(., t_{0}\right)\right\|_{2}^{2} \\
& \geq \frac{p-2}{2 p} K^{-1}\left\|w\left(., t_{0}\right)\right\|_{2}^{2},
\end{aligned}
$$

which contradicts (41). This completes the proof.

We are now ready to prove the finite time blow-up of solution to (1) when $\mathscr{E}(0)>0$.

Definition 2. We say that the function $w(x, t)$ blows up in finite time if there exists $t^{*} \in(0, \infty)$ such that

$$
\|w(x, t)\|_{L^{2}(\Omega)} \longrightarrow \infty \text { as } t \longrightarrow t^{*} .
$$

Theorem 1. Let $w_{0} \in W_{0}^{r, 2}(\Omega)$ and $w_{1} \in L^{2}(\Omega)$. Assume that $w_{0} \in \mathscr{M}, \mathscr{E}(0)>0$, and $\int_{\Omega} w_{0} w_{1} d x>0$, then any solution of (1) blows-up in finite time.

Proof. We have $w \in \mathscr{M}$; arguing by contradiction, we suppose that $w$ is weak global solution, for any $t \in[0, \infty)$. From (34) and Cauchy-Schwarts inequality, we get

$$
M^{\prime 2}(t)=4\left(w, \partial_{t}\right)^{2} \leq\|w\|^{2}\left\|\partial_{t} w\right\|^{2}, \quad t \in[0, \infty),
$$

which together with (36) implies that

$$
\begin{aligned}
& M^{\prime \prime}(t) M(t)-(1+\alpha)\left(M^{\prime}(t)\right)^{2} \\
& \geq\left(2\left\|\partial_{t} w\right\|^{2}-2\|w\|_{\substack{r, 2 \\
0}}^{2}+2 \int_{\Omega} w|w|^{p-2} w d x\right) M(t)-4(1+\alpha)\left(w, \partial_{t} w\right)^{2}
\end{aligned}
$$

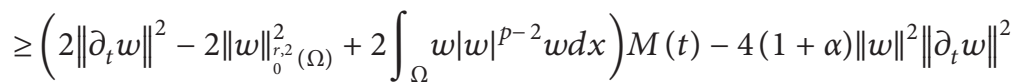

$$
\begin{aligned}
& =\left(-2\|w\|_{0}^{\|_{0}(\Omega)}{ }^{2}+2 \int_{\Omega} w|w|^{p-2} w d x-2(1+2 \alpha)\left\|\partial_{t} w\right\|^{2}\right) M(t)=A(t) M(t),
\end{aligned}
$$

where $\alpha>0$. We notice that

$$
\begin{aligned}
A(t) & :=-2\|w\|_{W_{0}^{r, 2}(\Omega)}^{2}+2\|w\|_{p}^{p}-2(1+2 \alpha)\left\|\partial_{t} w\right\|^{2} \\
& \geq-2(1+2 \alpha)\left\|\partial_{t} w\right\|^{2}-2\|w\|_{W_{0}^{r, 2}(\Omega)}^{2}+p\left\|\partial_{t} w\right\|^{2}+p\|w\|_{W_{0}^{r, 2}(\Omega)}^{2}-2 p \mathrm{E}(0) \\
& =-(4 \alpha-p+2)\left\|\partial_{t} w\right\|^{2}+(p-2)\|w\|_{W_{0}^{r, 2}(\Omega)}^{2}-2 p \mathrm{E}(0) \\
& \geq-(4 \alpha-p+2)\left\|\partial_{t} w\right\|^{2}+(p-2) K^{-1}\|w\|_{2}^{2}-2 p \mathrm{E}(0)
\end{aligned}
$$


for $t \in[t, \infty)$. Set $\alpha=(p-2) / 4>0$, and then $(4 \alpha-p+$ 2) $\left\|\partial_{t} w\right\|^{2}=0$. So from (47), we get

$$
A(t) \geq K^{-1}(p-2)\|w\|^{2}-2 p \mathscr{E}(0) \geq 0 .
$$

At this point, by (33)-(48), we obtain

$$
M^{\prime \prime}(t) M(t)-(1+\alpha)\left(M^{\prime}(t)\right)^{2}>0, \quad t \in[0, \infty),
$$

where $\alpha>0$. This implies that

$$
\begin{aligned}
& \left(M^{-\alpha}\right)^{\prime}=-\alpha M^{-\alpha-1} M^{\prime}(t)<0, \\
& \left(M^{-\alpha}\right)^{\prime \prime}=-\alpha M^{-\alpha-2}\left(M^{\prime \prime}(t) M(t)-(1+\alpha)\left(M^{\prime}(t)\right)^{2}\right)<0,
\end{aligned}
$$

for all $t \in[0, \infty)$, which means that the function $M^{-\alpha}$ is concave. Obviously, $M(0)>0$; then, there must exist a $T>0$ such that

$$
\lim _{t \rightarrow T M^{-a}}(t)=0
$$

so that

$$
\lim _{t \longrightarrow T^{-}} M(t)=\infty \quad \text { i.e., } \lim _{t \longrightarrow T^{-}}\|w\|^{2}=\infty \text {. }
$$

Thus, the proof is completed.

\section{Data Availability}

No data were used in this study.

\section{Conflicts of Interest}

The authors declare that they have no conflicts of interest.

\section{Authors' Contributions}

The authors contributed equally and significantly in writing this paper. All authors read and approved the final manuscript.

\section{References}

[1] G. H. Pertz and C. J. Gerhardt, Eds., Dritte Folge Mathematik (Erster Band). A. Asher \& Comp., Briefwechsel zwischen Leibniz, pp. 301-302, Hugens van Zulichem und dem Marquis de l'Hospital, 1849.

[2] L. Euler, "De progressionibus transcendendibus seu quarum termini generales algebraicae Dari nequent," Commentarii Academiae Scientiarum Imperialis Petropolitanae, 1730.

[3] S. F. Lacroix, Traite Du Calcul Differential et du Calcul Integral, Courcier, vol. 3, pp. 409-410, Paris, 2nd. edition, 1819.

[4] A. A. Kilbas, H. M. Srivastava, and J. J. Trujillo, "Theory and applications of fractional differential equations," Edited by J. V. Mill, Ed., Elsevier, Amsterdam, Netherlands, 2006.

[5] K. S. Miller and B. Ross, An Introduction to the Fractional Calculus and Fractional Deferential Equations, John Wiley \& Sons, New York, NY, USA, 1993.

[6] K. B. Oldham and J. Spanier, The Fractional Calculus, Academic Press, New York, NY, USA, 1974.

[7] L. M. Del Pezzo and J. D. Rossi, "Traces for fractional Sobolev spaces with variable exponents," Advances in Operator Theory, vol. 2, pp. 435-446, 2017.
[8] Y. Fu and P. Pucci, "On solutions of space-fractional diffusion equations by means of potential wells," Electronic Journal of Qualitative Theory of Differential Equations, vol. 70, 2016.

[9] Q. Li and L. He, "General decay and blow-up of solutions for a nonlinear viscoelastic wave equation with strong damping," Boundary Value Problems, vol. 153, 2018.

[10] M. Nakao, "Asymptotic stability of the bounded or almost periodic solution of the wave equation with nonlinear dissipative term," Journal of Mathematical Analysis and Applications, vol. 58, no. 2, pp. 336-343, 1977.

[11] D. G. Prakasha, N. S. Malagi, and P. Veeresha, "New approach for fractional schrödinger-boussinesq equations with mittagleffler kernel," Mathematical Methods in the Applied Sciences, vol. 43, no. 17, pp. 9654-9670, 2020.

[12] R. Servadei, E. Valdinoci, and E. Valdinoci, "Variational methods for non-local operators of elliptic type," Discrete \& Continuous Dynamical Systems - A, vol. 33, no. 5, pp. 21052137, 2013.

[13] J. L. Shomberg, "Well-posedness of semilinear strongly damped wave equations with fractional diffusion operators and $C^{0}$ potentials on arbitrary bounded domains," Rocky Mountain Journal of Mathematics, vol. 49, no. 4, pp. 13071334, 2019.

[14] D. G. Prakasha, N. S. Malagi, P. Veeresha, and B. C. Prasannakumara, "An efficient computational technique for time-fractional Kaup-Kupershmidt equation," Numerical Methods for Partial Differential Equations, vol. 37, no. 2, pp. 1299-1316, 2021.

[15] N. S. Malagi, P. Veeresha, B. C. Prasannakumara, G. D. Prasanna, and D. G. Prakasha, "A new computational technique for the analytic treatment of time-fractional Emden-Fowler equations," Mathematics and Computers in Simulation, vol. 190, pp. 362-376, 2021.

[16] N. Pan, P. Pucci, and B. Zhang, "Degenerate Kirchhoff-type hyperbolic problems involving the fractional Laplacian," Journal of Evolution Equations, vol. 18, no. 2, pp. 385-409, 2018.

[17] N. Pan, P. Pucci, R. Xu, and B. Zhang, "Degenerate Kirchhofftype wave problems involving the fractional Laplacian with nonlinear damping and source terms," Journal of Evolution Equations, vol. 19, no. 3, pp. 615-643, 2019.

[18] Q. Lin, X. T. Tian, X. Xu, and M. Zhang, "Blow up and blow up time for degenerate Kirchhoff-type wave problems involving the fractional Laplacian with arbitrary positive initial energy," Discrete \& Continuous Dynamical Systems - S, vol. 13, no. 7, pp. 2095-2107, 2020.

[19] E. Azroul, A. Benkirane, and M. Shimi, "Eigenvalue problem involving the fractional $p$-Laplacian operator," Advances in Operator Theory, vol. 4-2, pp. 539-555, 2009.

[20] A. Benaissa, D. Ouchenane, and Kh. Zennir, "Blow up of positive initial-energy solutions to systems of nonlinear wave equations with degenerate damping and source terms," Nonclinical Studies, (J. I), vol. 19, no. 4, pp. 523-535, 2012.

[21] A. Braik, Y. Miloudi, and K. Zennir, "A finite-time Blow-up result for a class of solutions with positive initial energy for coupled system of heat equations with memories," Mathematical Methods in the Applied Sciences, vol. 41, no. 4, pp. 1674-1682, 2018.

[22] U. Kaufmann, J. D. Rossi, and R. Vida, "Fractional Sobolev spaces with variables exponent and fractional $p(x)$-Laplacian, Electron," Journal of Qualitative Theory of Differential Equations, vol. 76, pp. 1-17, 2017. 
[23] N. I. Karachalios and N. M. Stavrakakis, "Global existence and blow-up results for some nonlinear wave equations on $\mathbb{R}^{N}$," Advances in Difference Equations, vol. 6, pp. 155-174, 2001.

[24] G. Liu and S. Xia, "Global existence and finite time blow up for a class of semilinear wave equations on $\mathrm{RN}$," Computers \& Mathematics with Applications, vol. 70, no. 6, pp. 1345-1356, 2015.

[25] K. zennir, "Growth of solutions with positive initial energy to system of degeneratly damped wave equations with memory," Lobachevskii Journal of Mathematics, vol. 35, no. 2, pp. 147156, 2014.

[26] kh. zennir, "Global nonexistence of solutions to system of nonlinear viscoelastic wave equations with degenerate damping and source terms," Ukrainian Mathematical Journal, vol. 65, no. 7, pp. 723-739, 2013.

[27] K. zennir and T. Miyasita, "Lifespan of solutions for a class of pseudo-parabolic equation with weak-memory," Alexandria Engineering Journal, vol. 59, no. 2, pp. 957-964, 2020.

[28] E. Di Nezza, G. Palatucci, and E. Valdinoci, "Hitchhiker's guide to the fractional Sobolev spaces," Bulletin des Sciences Mathematiques, vol. 136, no. 5, pp. 521-573, 2012. 